\title{
Widening and distortion of the particle energy distribution by chromaticity in quasi-isochronous rings
}

\author{
X. J. Deng $\odot,{ }^{1,}{ }^{*}$ R. Klein, ${ }^{2}$ A. W. Chao, ${ }^{3,4}$ A. Hoehl, ${ }^{2}$ W. H. Huang, ${ }^{1}$ J. Li®®, ${ }_{5}^{5}$ J. Lubeck, ${ }^{2}$ \\ Y. Petenev $\odot,{ }^{5}$ M. Ries, ${ }^{5}$ I. Seiler, ${ }^{5}$ C. X. Tang, ${ }^{1}$ and J. Feikes $\circledast^{5, \dagger}$ \\ ${ }^{1}$ Department of Engineering Physics, Tsinghua University, Beijing 100084, China \\ ${ }^{2}$ Physikalisch-Technische Bundesanstalt (PTB), Abbestraße 2-12, 10587 Berlin, Germany \\ ${ }^{3}$ Institute for Advanced Study, Tsinghua University, Beijing 100084, China \\ ${ }^{4}$ SLAC National Accelerator Laboratory, Stanford University, Stanford, California 94309, USA \\ ${ }^{5}$ Helmholtz-Zentrum Berlin (HZB), Albert-Einstein-Straße 15, 12489 Berlin, Germany
}

(Received 22 November 2019; accepted 1 April 2020; published 30 April 2020)

\begin{abstract}
This paper reports the observation of beam energy widening and distortion from Gaussian due to a nonvanishing horizontal chromaticity at a quasi-isochronous storage ring. The result originates from an average path-length dependence on the betatron oscillation amplitudes, which is intimately correlated to the transverse chromaticities. It is the first experimental validation of the impact of such a nonlinear transverselongitudinal coupling effect on the equilibrium beam characteristics in a storage ring. The results could be important for quasi-isochronous rings, steady-state microbunching, nonscaling fixed-field alternate gradient accelerators, etc., where very small momentum compaction or large chromaticity is required.
\end{abstract}

DOI: $10.1103 /$ PhysRevAccelBeams.23.044001

Quasi-isochronous (low-momentum-compaction) operation of an electron storage ring can be used to coherently enhance the synchrotron radiation at the wavelength of the resulting short bunch length (THz, Infrared), generate short $\mathrm{x}$-ray pulses, or maintain substructures on the bunch generated by external impedances or actively applied fields over many turns. Understanding and controlling the dynamics at low momentum compaction is important also for the present accelerator upgrades and advances, the general trend of diffraction-limited storage ring design and the novel mechanisms like steady-state microbunching (SSMB) [1-3]. As the ring is pushed from the well-known type of operation at higher momentum compaction close to the isochronous point, the beam dynamics changes drastically and many classical formulas and scaling laws for beam parameters break down.

From the single-particle dynamics perspective, there are several bunch lengthening and energy broadening effects [4-7]. Among them a fundamental one is the second-order transverse-longitudinal coupling. This effect originates from an average path-length dependence on the betatron oscillation amplitudes, which can be expressed concisely by the formula

\footnotetext{
*dxj11@tsinghua.org.cn

joerg.feikes@helmholtz-berlin.de
}

Published by the American Physical Society under the terms of the Creative Commons Attribution 4.0 International license. Further distribution of this work must maintain attribution to the author(s) and the published article's title, journal citation, and DOI.

$$
\Delta C=-2 \pi\left(\xi_{x} J_{x}+\xi_{y} J_{y}\right)
$$

as a result of symplecticity of Hamiltonian dynamics [8], in which $\Delta C$ is the average path-length deviation relative to the ideal particle, $\xi_{x, y}$ and $J_{x, y}$ are the horizontal (vertical) chromaticity and betatron action, respectively. The manifestation and importance of this effect under different contexts have been theoretically analyzed by several authors before [6-14]. Recently, there are two experiments $[15,16]$ confirming the relation in Eq. (1). In this paper, we report the first experimental investigation of the influence of this effect on the equilibrium beam characteristics in storage rings. It has been proved that a nonvanishing horizontal chromaticity broadens the energy spread and distorts the beam from Gaussian in a quasi-isochronous ring. The experiment is conducted at the electron storage ring, the Metrology Light Source (MLS) [17,18], of the Physikalisch-Technische Bundesanstalt in Berlin.

Due to this effect, particles with different betatron amplitudes lose synchronization with each other when traversing a lattice with nonzero chromaticity. In a storage ring, however, the rf cavity makes all the particles synchronize with it automatically in an average sense. It accomplishes this by introducing a betatron-amplitudedependent momentum shift to compensate the path-length difference [6]

$$
\Delta \delta=-\frac{\Delta C}{\alpha C_{0}},
$$


where $\Delta \delta$ is the shift of relative momentum deviation $\delta=d p / p_{0}, \alpha$ is the momentum compaction factor of the ring and $C_{0}$ is the ring circumference. This shift results in beam energy widening in a low-momentum-compaction ring with nonzero chromaticity as different particles have different betatron actions.

For an electron storage ring, when the quantum excitation is also included, the total relative momentum deviation of a particle is

$$
\delta=\Delta \delta+\delta_{\mathrm{qe}},
$$

in which $\delta_{\text {qe }}$ represents the quantum excitation contribution. Since the vertical emittance is much smaller than the horizontal one in planar rings, we consider only the impact from the horizontal emittance. When the coupling is not very strong, the distribution of $J_{x}$ and $\delta_{\mathrm{qe}}$ can still be approximated by an exponential and a Gaussian distribution, respectively, and independent of each other,

$$
\psi\left(J_{x}\right)=\frac{1}{2 \pi \epsilon_{x 0}} e^{-\frac{J_{x}}{\epsilon_{x 0}}}, \quad \psi\left(\delta_{\mathrm{qe}}\right)=\frac{1}{\sqrt{2 \pi} \sigma_{\delta 0}} e^{-\frac{\delta_{\mathrm{qe}}^{2}}{2 \sigma_{\delta 0}^{2}}},
$$

with $\epsilon_{x 0}$ and $\sigma_{\delta 0}$ being the natural horizontal emittance and energy spread from quantum radiation [19]. The distribution of $\delta$ is then an exponentially modified Gaussian [7]

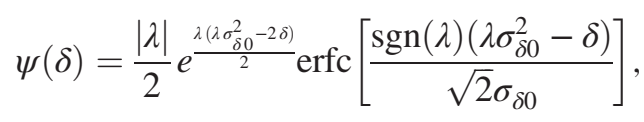

where $\lambda=\frac{\alpha C_{0}}{2 \pi \xi_{\xi_{x}} x_{00}}, \operatorname{sgn}(x)$ is the sign function and $\operatorname{erfc}(x)=$ $1-\operatorname{erf}(x)=\frac{2}{\sqrt{\pi}} \int_{x}^{\infty} e^{-t^{2}} d t$ is the complementary error function. Which side leans and how long the tail of the non-Gaussian distribution is, depend on the signs and magnitudes of $\alpha, \xi_{x}$ and the values of $\epsilon_{x 0}, \sigma_{\delta 0}$. The variance of $\delta$ is therefore

$$
\sigma_{\delta}^{2}=\sigma_{\delta 0}^{2}+\left(\frac{2 \pi \epsilon_{x 0} \xi_{x}}{\alpha C_{0}}\right)^{2}
$$

The energy spread grows with the magnitude decrease of $\alpha$ when $\xi_{x} \neq 0$.

Putting in the typical low momentum compaction of $\alpha=$ $1 \times 10^{-4}$ and the other operating parameters shown in Tab. I for the MLS, the energy spread brought by this coupling effect at $\xi_{x}=1$ can be as significant as its natural value. At the MLS, the Compton-backscattering (CBS) method is applied to measure the electron energy [20]. Nevertheless, within certain limitations, the electron beam energy spread can also be evaluated from the CBS photon spectra [21]. The non-Gaussian momentum distribution makes the evaluation a bit more involved, but we can assume a Gaussian distribution with an equivalent mean energy spread. This is a good approximation as long as $\xi_{x}$ is not too large.
TABLE I. Parameters of the MLS in the experiment.

\begin{tabular}{lcl}
\hline \hline Parameter & Value & \multicolumn{1}{c}{ Description } \\
\hline$C_{0}$ & $48 \mathrm{~m}$ & Ring circumference \\
$E_{0}$ & $630 \mathrm{MeV}$ & Beam energy \\
$f_{\mathrm{rf}}$ & $500 \mathrm{MHz}$ & rf frequency \\
$V_{\mathrm{rf}}$ & $\leq 600 \mathrm{kV}$ & rf voltage \\
$\epsilon_{x 0}$ & $250 \mathrm{~nm}$ & Horizontal emittance \\
$\sigma_{\delta 0}$ & $4.4 \times 10^{-4}$ & Natural energy spread \\
\hline \hline
\end{tabular}

The experiment is conducted with all 80 rf buckets equally filled. To exclude a severe impact from energy widening collective effects, the beam current is decayed till the horizontal beam size is not sensitively dependent on it. The average single bunch current is below $12.5 \mu \mathrm{A}$ $\left(1 \times 10^{6}\right.$ electrons $\left./ 1 \mu \mathrm{A}\right)$ while doing the CBS measurements. To mitigate the influence of a nonlinear momentum compaction, the longitudinal chromaticity has been corrected close to zero. The other parameters of the ring in the experiment are presented in Table I.

To get the energy spread based on the CBS method with precision, the HPGe-detector used in the measurement should be calibrated in terms of photon energy per channel. This is realized by recording the emission lines from a ${ }^{152} \mathrm{Eu}$ radionuclide simultaneously during the measurement of the CBS photons. Moreover, the width of the fitted ${ }^{152} \mathrm{Eu}$ lines that are close to the edge of the CBS photons have been used to determine the detector resolution $\sigma_{\mathrm{det}}$ at the photon energy of the CBS cutoff edge, $E_{\text {edge }}$, in our case $707 \mathrm{keV}$. This is done by a linear interpolation of the width of the ${ }^{152} \mathrm{Eu}$ lines at $344.28 \mathrm{keV}$ and $778.90 \mathrm{keV}$. The detector resolution $\sigma_{\text {det }}$ at $707 \mathrm{keV}$ is thus determined to be 0.64(4) $\mathrm{keV}$.

Figure 1(a) shows the typical CBS photon spectra close to the cutoff edge under the cases of different $\xi_{x}$ and $\alpha$. The adjustment of $\xi_{x}$ is accomplished by the implementation of different chromatic sextupole strengths and the $\alpha$ by slightly tuning quadrupole strengths. In the experiment, the rf voltage is kept constant and the synchrotron frequency $f_{s}$ is proportional to the square root of the magnitude of $\alpha$. The edge in the figure is a convolution of a step function representing the CBS cutoff edge with a Gaussian function which attributes to the finite HPGe-detector energy resolution and the electron beam energy spread. The fitted line is basically an error function from which the energy width of the CBS photons at the edge $\sigma_{\text {edge }}$, and therefore the electron beam energy spread $\sigma_{\delta}$, can be deduced. It is assumed $\sigma_{\text {edge }}$ is given by $\sigma_{\text {edge }}=\sqrt{\sigma_{\text {det }}^{2}+\left(2 E_{\text {edge }} \sigma_{\delta}\right)^{2}}$. The second term in the square root is due to the electron beam energy spread, and is based on the fact that the energy of the backscattered photon is proportional to the electron energy squared.

It can be seen from Fig. 1(a) that the edge slope decreases with the magnitude increasing of $\xi_{x}$ and lowering of $\alpha$ when 

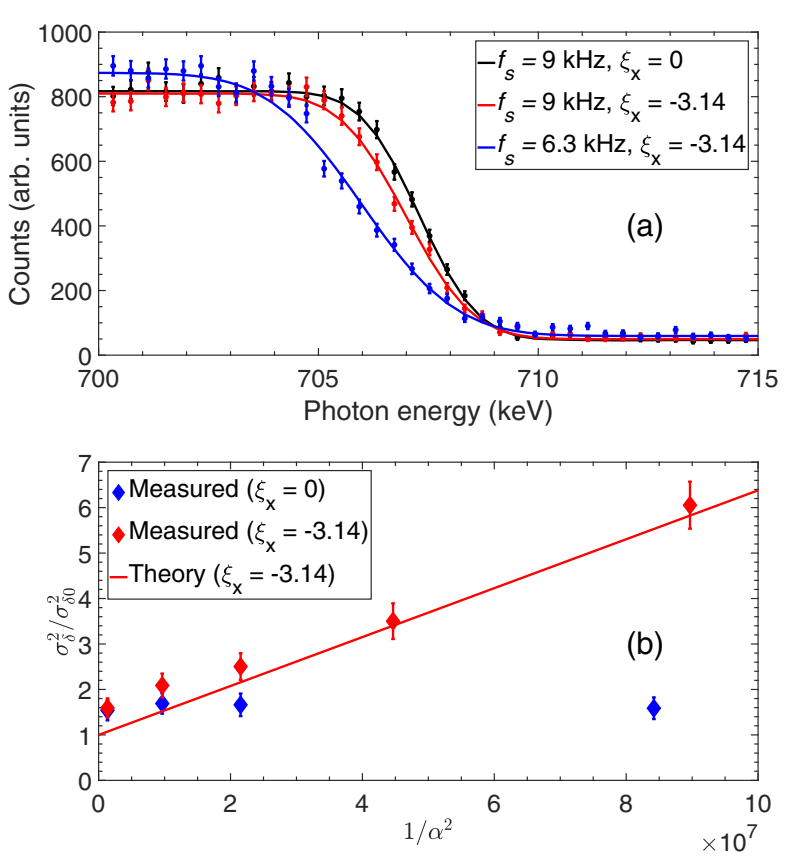

FIG. 1. Measurement of the electron beam energy widening brought by the horizontal chromaticity using the CBS method. (a) The cutoff edges of CBS photon spectra under different $\xi_{x}$ and $f_{s}$ (therefore $\alpha$ ). (b) Quantitative evaluation of the cut-off edges revealing the energy spread and its comparison with theory Eq. (6). The error bars in both figures are the one sigma uncertainties of the measurements and are due to calibration errors and counting statistics. The data acquisition of each spectrum takes 15 minutes.

$\xi_{x} \neq 0$, which indicates that there is an energy widening in the process. Quantitative evaluation of the edges revealing the energy spread and its comparison with theory of Eq. (6) are shown in Fig. 1(b). The energy spread grows significantly with the magnitude decrease of $\alpha$ when $\xi_{x}=-3.14$, while it stays almost constant in the $\xi_{x}=0$ case. The agreement between measurements and theory is quite satisfactory. This is the first direct experimental proof of the impact of this effect on the equilibrium beam parameters in a storage ring.

As discussed in [6], a shift of the momentum center corresponds to a shift of the synchronous rf phase, so particles with different betatron amplitudes will oscillate around different fixed points in the longitudinal dimension, thus lengthening the bunch. However, this lengthening of bunch is much less notable compared to the energy widening at the MLS [7]. Also due to the limited resolution of the present streak camera, we do not measure the bunch lengthening in the experiment. Nevertheless, a more comprehensive investigation of this effect can be conducted on the other beam characteristics, like the transverse intensity distribution.

The horizontal position of a particle is

$$
x=\sqrt{2 J_{x} \beta_{x}} \cos \varphi_{x}+D_{x}\left(\delta_{\mathrm{qe}}+\frac{2 \pi \xi_{x} J_{x}}{\alpha C_{0}}\right),
$$

in which $D_{x}, \beta_{x}$, and $\varphi_{x}$ are the horizontal dispersion, beta function and betatron phase, respectively. The concept of Courant-Snyder functions is assumed to be still valid when the coupling is not very strong in Eq. (7). It can be seen from the equation that particles with different betatron amplitudes oscillate around different closed orbits, which is the amplitude-dependent center shift [15]. Because of the dispersion, the non-Gaussian particle momentum distribution can also be reflected to the transverse dimension, which can be observed by the beam imaging systems installed at the MLS [22].

Figure 2 shows the typical transverse beam intensity distribution measured by the imaging systems at two dispersive locations, QPD0 and QPD1, with different values of $\xi_{x}$ in both the negative and positive-momentum-compaction modes. The relevant optics functions, $\beta_{x}$ and $D_{x}$, at the two observation points are also shown in the figure. Note $D_{x}$ have different signs and magnitudes at QPD0 and QPD1. It can be seen that the horizontal beam distribution at these dispersive locations becomes asymmetric when $\xi_{x} \neq 0$. The long tail direction and the magnitude of deviation from Gaussian are determined by the signs and magnitudes of $\alpha, D_{x}, \xi_{x}$ and also the values of $\epsilon_{x 0}$ and $\beta_{x}$, which fits with the expectations.

Figure 3(a) demonstrates the typical horizontal beam profiles measured at QPD1 in the negative-momentumcompaction mode under three different $\xi_{x}$ and their good agreements with theory. It turns out that both the theoretical and experimental measured horizontal coordinate distribution $\psi(x)$ can be excellently fitted by an exponentially modified Gaussian function

$$
\psi(x)=\frac{1}{\sqrt{2 \pi} \sigma} \cdot e^{-\frac{(x-b)^{2}}{2 \sigma^{2}}} \cdot\left(1+\operatorname{erf}\left[d \cdot \frac{x-b}{\sqrt{2} \sigma}\right]\right) .
$$

The reason is that the distribution of particle momentum is approximately an exponentially modified Gaussian as analyzed above. The asymmetry parameter $d$ in Eq. (8) is used to quantitatively describe the deviation from Gaussian and as a criterion to do comparison between measurements and theory. Figures 3(b) and (c) show the asymmetry parameter $d$ versus $\xi_{x}$ and $f_{s}$, therefore $\alpha$, from measurements and theory at QPD0 and QPD1. It can be seen that the larger the $\xi_{x}$ and the smaller the $\alpha$, the more asymmetric the distribution is. Also the asymmetry at QPD1 is more significant than that at QPD0, as the magnitude of $D_{x}$ at QPD1 is larger while the $\beta_{x}$ difference at two places is not much. The agreement between measurements and theory confirms that this effect distorts the beam from Gaussian in both the longitudinal and transverse dimensions.

While the energy widening and beam distortion could be a detrimental outcome for some applications, it may actually also be beneficial as it can help to stabilize collective instabilities. The bunch lengthening on the other hand is much less notable compared to the energy widening. So quasi-isochronous ring-based coherent radiation 


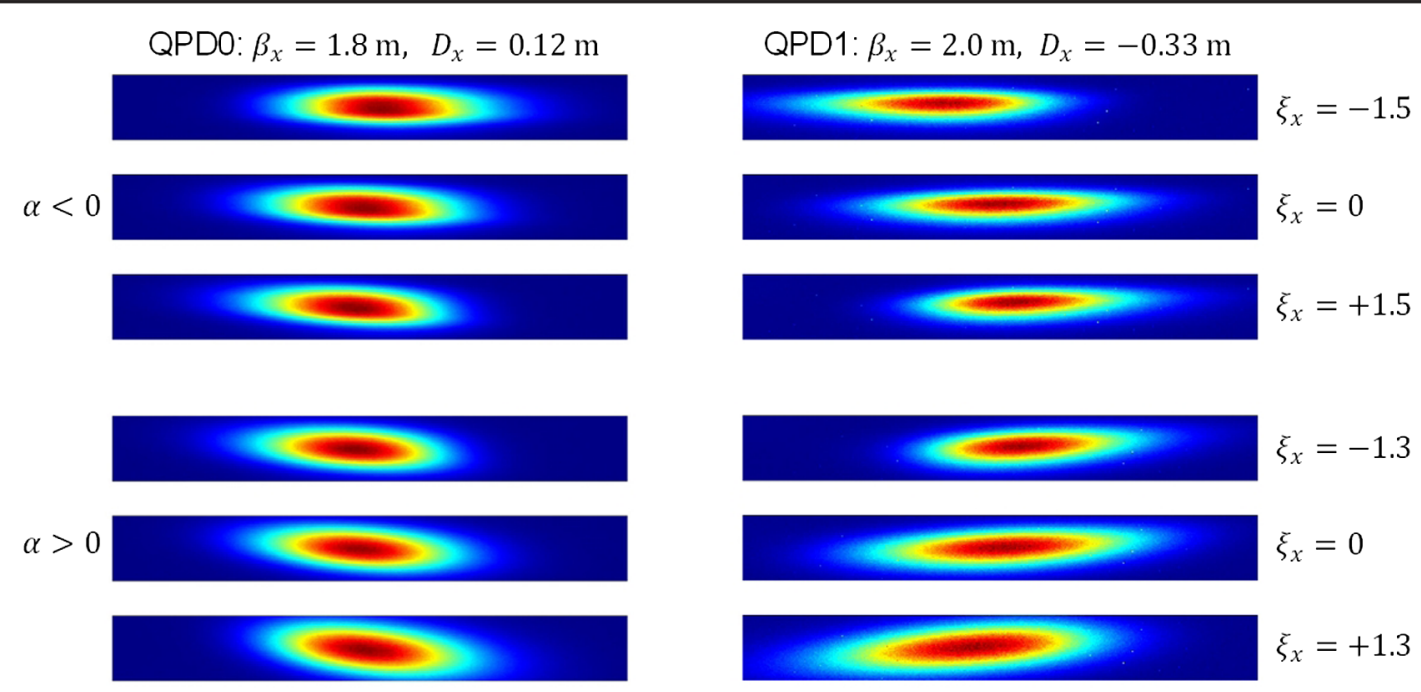

FIG. 2. Transverse beam intensity distortion from Gaussian at dispersive locations due to a nonvanishing horizontal chromaticity measured by the imaging systems installed at QPD0 and QPD1. Three different $\xi_{x}$ are applied in both the negative and positivemomentum-compaction modes with $\alpha= \pm 8.4 \times 10^{-5}$. There is some residual horizontal-vertical coupling in the positive-momentumcompaction case, which do not influence the principle observation of the non-Gaussian behavior.

schemes, like some of the SSMB scenarios [1-3], may boost the stable coherent radiation power by taking advantage of this effect. For example, the stable single bunch current at the MLS can grow for more than one order of magnitude by increasing the absolute value of the horizontal chromaticity from zero to a value larger than three, with the head-tail and the other collective effects like the longitudinal microwave instability properly suppressed. It has been proved at the MLS that the increase of $\mathrm{THz}$ power due to a higher stable beam current overcompensates the decrease due to the slight bunch lengthening of this effect. Therefore, this is now the standard low-momentumcompaction mode at the MLS for the application of Fourier transform spectroscopy [23].
This effect may also be relevant for the momentum and dynamic aperture in storage rings $[14,24]$, for instance, due to the large betatron amplitude induced by Touschek scattering or the large natural chromaticity in the low-emittance lattice design. Another example where this effect could be crucial is the nonscaling fixed-field alternate gradient (FFAG) accelerators for muon acceleration [13]. A linear nonscaling FFAG has its natural chromaticity uncorrected to achieve a large transverse acceptance as the emittance of the muon beam is usually very large. The energy spread grows significantly after traversing the acceleration lattice. Solutions similar to the beam conditioning [10] for free-electron lasers (FELs) in the $\mathrm{x}$-ray regime can be invoked. Investigations presented in this paper can give clues for new beam conditioning ideas.
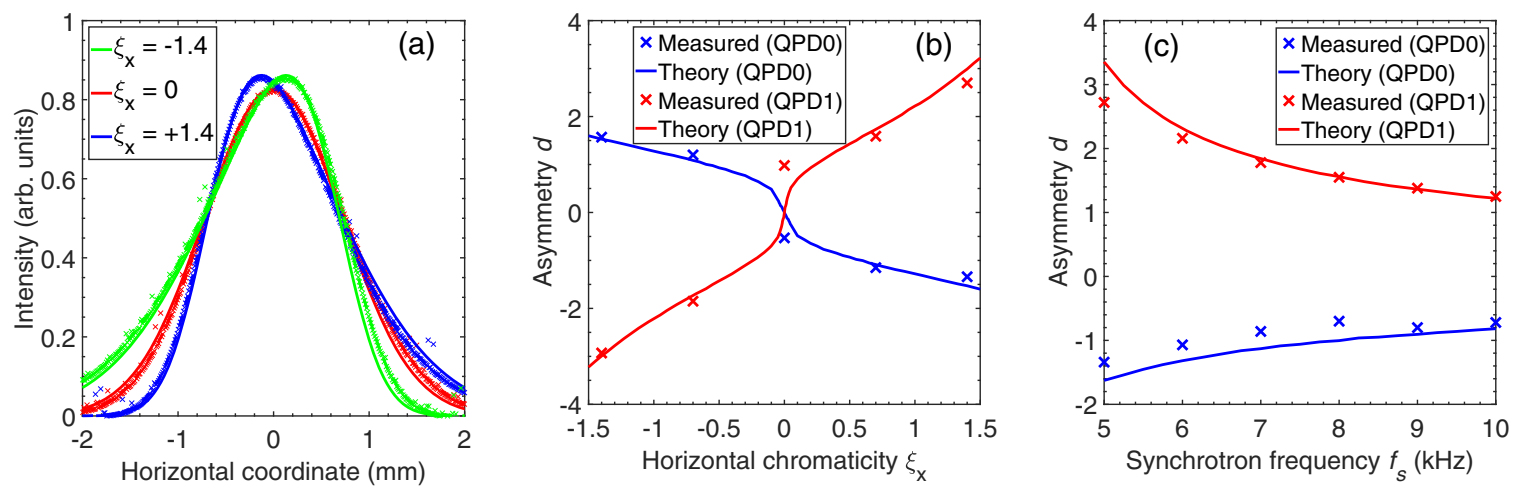

FIG. 3. Horizontal beam profile distortion from Gaussian by horizontal chromaticity. (a) Typical horizontal beam profile at QPD1 with $\alpha=-7 \times 10^{-5}$ under three different $\xi_{x}$. The closed-orbit movements of the ideal particle due to the sextupole strengths changes when adjusting $\xi_{x}$ have been compensated in the plot. Cross: beam imaging system measurement results. Dashed line: fit of the measurement data by an exponentially modified Gaussian function Eq. (8). Solid line: theoretical prediction. (b) Measured and theoretical asymmetry parameter $d$ versus $\xi_{x}$ at QPD0 and QPD1 with $f_{s}=5 \mathrm{kHz}\left(\alpha=-7 \times 10^{-5}\right)$; (c) Measured and theoretical asymmetry parameter $d$ versus $f_{s}$ at QPD0 and QPD1 with $\xi_{x}=1.4$. All the theoretical curves are obtained based on Eq. (4) and Eq. (7). 
For example, an upstream transport lattice with an opposite chromaticity to the natural chromaticity of the FEL undulator or nonscaling FFAG can mitigate the influence of this effect.

This nonlinear transverse-longitudinal coupling may also be useful in some more applications. For example, it can be used for the real-time emittance evaluation on storage rings if the chromaticities, beta function, and dispersion are known, which are usually easier to get than measuring the emittance directly. The amplitude-dependent center shift can be applied to detect beam instabilities which blow up the transverse emittance [25]. A strongly asymmetric particle momentum distribution due to this effect cooperating with a large-momentum-compaction lattice can generate a strongly asymmetric distributed current, which is favored in some applications such as beam driven wakefield acceleration [26].

\section{ACKNOWLEDGMENTS}

We thank our HZB, PTB and Tsinghua colleagues who help to make what is presented in this paper possible. The work of Deng, Huang and Tang is supported by the Tsinghua University Initiative Scientific Research Program No. 20191081195, China.

[1] D. F. Ratner and A. W. Chao, Steady-State Microbunching in a Storage Ring for Generating Coherent Radiation, Phys. Rev. Lett. 105, 154801 (2010).

[2] A. Chao, E. Granados, X. Huang, D. Ratner, and H.-W. Luo, High power radiation sources using the steady-state microbunching mechanism, in Proceedings of the 7 th International Particle Accelerator Conference (IPAC'16), Busan, Korea, 2016 (JACoW, Geneva, 2016), pp. 10481053.

[3] C. Tang, X. Deng, A. Chao, W. Huang, T. Rui, J. Feikes, J. Li, M. Ries, A. Hoehl, D. Ratner et al., An overview of the progress on SSMB, in Proceedings of the 60th ICFA Advanced Beam Dynamics Workshop on Future Light Sources (FLS'18), Shanghai, China, 2018 (JACoW, Geneva, 2018), pp. 166-170.

[4] Y. Shoji, H. Tanaka, M. Takao, and K. Soutome, Longitudinal radiation excitation in an electron storage ring, Phys. Rev. E 54, R4556 (1996).

[5] Y. Shoji, Bunch lengthening by a betatron motion in quasiisochronous storage rings, Phys. Rev. Accel. Beams 7, 090703 (2004).

[6] Y. Shoji, Dependence of average path length betatron motion in a storage ring, Phys. Rev. Accel. Beams 8, 094001 (2005).

[7] X. J. Deng, A. W. Chao, J. Feikes, W. H. Huang, M. Ries, and C. X. Tang, Single-particle dynamics of microbunching, Phys. Rev. Accel. Beams 23, 044002 (2020).

[8] É. Forest, Beam Dynamics: A New Attitude and Framework (Harwood Academic Publisher, Amsterdam, 1998), Chap. 9, p. 264.

[9] A. Artamonov, I. S. Derbenev, and N. Inozemtsev, The possibility of controlling the dispersion characteristics of particle motion in an undulator, Zh. Tekh. Fiz. 59, 214 (1989).

[10] A. M. Sessler, D. H. Whittum, and L.-H. Yu, RadioFrequency Beam Conditioner for Fast-Wave Free-Electron Generators of Coherent Radiation, Phys. Rev. Lett. 68, 309 (1992).

[11] L. Emery, Coupling of betatron motion to the longitudinal plane through path lengthening in low alpha(c) storage rings, in Proceedings of the 15th International Conference on High Energy Accelerators, edited by J. Rossbach (World Scientific, Singapore, 1993), pp. 1172-1174.

[12] A. Chao, Lecture notes on topics in accelerator physics, Technical Report No. SLAC-PUB-9574, 2002.

[13] J. S. Berg, Amplitude dependence of time of flight and its connection to chromaticity, Nucl. Instrum. Methods Phys. Res., Sect. A 570, 15 (2007).

[14] M. Takao, Impact of betatron motion on path lengthening and momentum aperture in a storage ring, in Proceedings of the 11th European Particle Accelerator Conference, Genoa, 2008 (EPS-AG, Genoa, Italy, 2008), pp. 3152-3154.

[15] Y. Shoji, M. Takao, and T. Nakamura, Amplitude dependent shift of betatron oscillation center, Phys. Rev. Accel. Beams 17, 064001 (2014).

[16] S. Machida, D. J. Kelliher, C. S. Edmonds, I. W. Kirkman, J. S. Berg, J. K. Jones, B. D. Muratori, and J. M. Garland, Amplitude-dependent orbital period in alternating gradient accelerators, Prog. Theor. Exp. Phys. 2016, 033 G01 (2016).

[17] J. Feikes, M. von Hartrott, M. Ries, P. Schmid, G. Wüstefeld, A. Hoehl, R. Klein, R. Müller, and G. Ulm, Metrology Light Source: The first electron storage ring optimized for generating coherent $\mathrm{THz}$ radiation, Phys. Rev. Accel. Beams 14, 030705 (2011).

[18] M. Ries, Nonlinear momentum compaction and coherent synchrotron radiation at the metrology light source, Dissertation, Humboldt University of Berlin, Berlin, Germany, 2014.

[19] M. Sands, The physics of electron storage rings: An introduction, Technical Report No. SLAC-121, 1970.

[20] R. Klein, G. Brandt, R. Fliegauf, A. Hoehl, R. Müller, R. Thornagel, G. Ulm, M. Abo-Bakr, J. Feikes, M. v. Hartrott, K. Holldack, and G. Wüstefeld, Operation of the Metrology Light Source as a primary radiation source standard, Phys. Rev. Accel. Beams 11, 110701 (2008).

[21] R. Klein, T. Mayer, P. Kuske, R. Thornagel, and G. Ulm, Beam diagnostics at the BESSY I electron storage ring with Compton backscattered laser photons: measurement of the electron energy and related quantities, Nucl. Instrum. Methods Phys. Res., Sect. A 384, 293 (1997).

[22] C. Koschitzki, A. Hoehl, R. Klein, R. Thornagel, J. Feikes, M. Hartrott, and G. Wüstefeld, Highly sensitive beam size monitor for pA currents at the MLS electron storage ring, in Proceedings of the 1st International Particle Accelerator Conference (IPAC'10), Kyoto, Japan, 2010 (JACoW, Geneva, 2010), pp. 894-896.

[23] J. Feikes and A. Hoehl (private communication).

[24] L. Hoummi, J. R. Lopez, C. Welsch, A. Loulergue, and R. Nagaoka, Beam dynamics in MBA lattices with different chromaticity correction schemes, in Proceedings of the 10th International Particle Accelerator Conference 
(IPAC'19), Melbourne, Australia, 2019 (JACoW, Geneva, 2019), pp. 346-349.

[25] K. Kobayashi, J. Schimizu, T. Hara, T. Seike, K. Soutome, M. Takao, and T. Nakamura, Amplitude dependent betatron oscillation center shift by non-linearity and beam instability interlock, in Proceedings of the 2nd
International Particle Accelerator Conference (IPAC'11), San Sebastián, Spain (2011), pp. 2178-2180.

[26] P. Chen, J. J. Su, J. M. Dawson, K. L. F. Bane, and P. B. Wilson, Energy Transfer in the Plasma Wake-Field Accelerator, Phys. Rev. Lett. 56, 1252 (1986). 